\title{
Anti-thyroid antibodies, parietal cell antibodies and tissue transglutaminase antibodies in patients with autoimmune thyroid disease
}

\author{
Orit Twito ${ }^{1}$, Yonatan Shapiro ${ }^{2}$, Aviva Golan-Cohen ${ }^{3,4}$, Yoav Dickstein ${ }^{3}$, Rosane Ness-Abramof ${ }^{5}$, \\ Menachem Shapiro ${ }^{3}$
}

\author{
${ }^{1}$ Meir Medical Center, Kefar-Sava, Israel \\ ${ }^{2}$ Maccabi Health Services, Israel \\ ${ }^{3}$ Leumit Health Fund, Israel \\ ${ }^{4}$ Department of Family Medicine, Tel-Aviv University, Tel-Aviv, Israel \\ ${ }^{5}$ Clalit Health Services, Israel
}

Submitted: 4 November 2015

Accepted: 30 December 2015

Arch Med Sci 2018; 14, 3: 516-520

DOI: https://doi.org/10.5114/aoms.2016.58743

Copyright @ 2016 termedia \& Banach

\begin{abstract}
Introduction: The co-existence of tissue-specific autoantibodies in autoimmune thyroid disease (ATD) is well established. The published prevalence of anti-parietal cell antibodies (PC-Ab) is $20-25 \%$, and that of celiac antibodies is $2-5 \%$. The goal of this study was to determine the prevalence of PC-Ab and anti-tissue transglutaminase antibodies (tTG-Ab) in patients with ATD and to evaluate the correlation between anti-thyroid antibodies and the other antibodies.

Material and methods: The files of 120 Israeli Jews and Arabs with ATD were evaluated for anti-thyroglobulin antibodies (Tg-Ab), anti-thyroid peroxidase antibodies (TPO-Ab), PC-Ab and tTG-Ab. For patients with positive PC-Ab and/or tTG-Ab, upper gastrointestinal (GI) endoscopy results were recorded. Gastrin levels were collected in patients with positive PC-Ab.

Results: Twelve (10\%) males and 108 (90\%) females were evaluated, of whom 93.33\% had Hashimoto's thyroiditis. Thirty-four (28.3\%) subjects had positive PC-Ab. This rate was not affected by gender, ethnicity or thyroid disease. Abnormal gastroscopy findings were documented in $95.2 \%$ of the upper Gl endoscopies. The mean gastrin level in this subgroup was $660.4 \mathrm{pg} / \mathrm{ml}$. Five of 114 tTG-Ab tests were positive (4.4\%). All were females with Hashimoto's thyroiditis. Rates were equal among Jews and Arabs. Higher TPO-Ab levels were associated with higher risk for PC-Ab positivity $(p=0.027)$, but not tTG-positivity. Higher Tg-Ab levels were not associated with higher levels of other antibodies.

Conclusions: Considering the frequency of PC-Ab and tTG-Ab positivity in ATD, checking for the presence of these two entities should be an integral part of the workup of this disease.
\end{abstract}

Key words: autoimmune thyroid disease, anti-thyroid antibodies, parietal cell antibodies, celiac disease.

\section{Introduction}

The association of autoimmune thyroid disease (ATD) with other autoimmune disorders is well established.

\author{
Corresponding author: \\ Orit Twito MD \\ Meir Medical Center \\ 59 Tchenichovsky St \\ 44281 Kefar-Sava, Israel \\ Phone: 972-9-7472788 \\ Fax: 972-9-7471644 \\ E-mail: orit.twito@clalit.org.il
}




\section{Autoimmune thyroid disease and autoimmune gastritis}

Anti-parietal cell antibodies (PC-Ab) are associated with the development of autoimmune gastritis, usually limited to the fundus and body of the stomach [1]. The presence of PC-Ab in patients with ATD predicts the development of autoimmune gastritis [2] and correlates with biopsy-proven fundal gastritis [3]. Autoimmune gastritis can lead to vitamin $B_{12}$ deficiency, iron deficiency anemia and, in rare cases, type I gastric neuroendocrine tumors $[1,4]$. The prevalence of PC-Ab in patients with ATD is $20-29 \%[2,5,6]$, and $28 \%$ have vitamin $B_{12}$ deficiency [7]. The prevalence of PC-Ab in subjects with ATD in Israel is unknown.

\section{Autoimmune thyroid disease and celiac disease}

The classic malabsorption syndrome presentation of celiac disease is not common in the adult population. Adult celiac disease usually presents with mild gastrointestinal symptoms, anemia or osteoporosis. Prompt treatment is associated with symptomatic improvement, reduced risk of malignancy and lower mortality rate [8]. Tissue transglutaminase (tTG) is a calcium-dependent enzyme that plays a crucial role in celiac disease pathogenesis. During gluten consumption anti-tissue transglutaminase antibodies (tTG-Ab) are produced by the mucosa of the small intestine. Immunoglobulin A (IgA) tTG-Ab has high sensitivity and specificity for the detection of celiac disease in adults [9]. The prevalence of celiac antibodies in patients with ATD is $2-5 \%[10,11]$ : much higher than the estimated prevalence in the general population, which is $0.3-1.4 \%$ in most countries [12]. The prevalence of tTG-Ab in patients with ATD in Israel is unknown.

The aim of this study was to determine the prevalence of PC-Ab and tTG-Ab in patients with ATD in a heterogeneous urban population in Israel and to identify the correlation between anti-thyroid antibody levels and the other antibodies tested.

\section{Material and methods}

\section{Patients and methods}

The files of 120 consecutive patients with ATD from a community endocrinology clinic were reviewed. These patients were diagnosed with either Hashimoto's thyroiditis or Graves' disease. Data collected included anti-thyroglobulin antibodies (Tg-Ab), anti-thyroid peroxidase antibodies (TPO$A b), P C-A b$ and tTG-Ab. For patients with positive PC-Ab and/or tTG-Ab, results of upper gastrointestinal (GI) endoscopy were recorded. Gastrin levels were collected in patients with positive PC-Ab.
Anti-thyroid peroxidase antibodies and Tg-Ab were measured using a solid phase, enzymelabeled, chemiluminescent sequential immunometric assay (Immulite 2000, Siemens Healthcare Diagnostics Inc., UK). TPO-Ab and Tg-Ab levels were expressed in IU/ml. IgA tTG-Ab were measured by multiplex flow immunoassay (Bioplex 2200, BioRad Laboratories, Inc.). Anti-tissue transglutaminase antibodies levels were measured in $\mathrm{U} / \mathrm{ml}$, and expressed by positivity. PC-Ab were measured by immunofluorescence antibody assay. Gastrin levels were measured by a chemiluminescent, enzyme-labeled immunometric assay (Immulite 2000, Siemens Healthcare Diagnostics Inc., UK). The normal reference range for serum gastrin is $13-115 \mathrm{pg} / \mathrm{ml}$.

\section{Ethical consideration}

This study was approved by the local institution review board. Informed consent was not required.

\section{Statistical analysis}

A binary logistic regression model was used to estimate the association between positive PC-Ab and demographic data in multivariate analyses. Interaction terms for statistically significant effect modifiers were added to the multivariate model when appropriate.

$T$-test for equality of means was used for comparison of the means of TPO-Ab and Tg-Ab titers. Statistical analysis was performed using SPSS software, version 20.

\section{Results}

\section{Patient population}

A total of 120 patients at a mean age of 50.4 \pm 16.1 years (range: $15-79$ years) were included in the study. Twelve (10\%) males and $108(90 \%)$ females were evaluated; 112 (93.3\%) had Hashimoto's thyroiditis and the other 8 (6.7\%) had Graves' disease. Twenty-four (20\%) patients were Arabs and 96 (80\%) were Jews.

\section{Incidence of positive PC-Ab in different groups}

Thirty-four (28.3\%) subjects had positive PC-Ab. The rate of positive PC-Ab was associated with older age (54.8 vs. $48.5, p<0.05)$, but not with gender $(33.3 \%$ in males vs. $28.7 \%$ in females, $p>0.05$ ), ethnicity ( $30.2 \%$ in Jews vs. $25 \%$ in Arabs, $p>0.05$ ) or thyroid disease (29.4\% in Hashimoto's thyroiditis vs. $25 \%$ in Graves' disease, $p>0.05$ ).

\section{Results of Gl endoscopy}

Twenty-six of the PC-Ab positive patients underwent an upper GI endoscopy, with abnormal 
results in all but 1 (95.5\%). Atrophic gastritis was documented in 19 (73.1\%) patients. Six (23.1\%) patients presented with intestinal metaplasia in addition to the atrophy, and 1 (3.8\%) had gastric neuroendocrine tumor type I on a background of atrophic gastritis. Six (23.1\%) patients were diagnosed with non-specific gastritis.

\section{Serum gastrin levels}

Gastrin serum levels were measured in 23 of the 34 patients with positive PC-Ab. Hypergastrinemia was documented in 18 (78.3\%). Mean gastrin level in this subgroup was $688.7 \mathrm{pg} / \mathrm{ml}$, ranging from 38 to $2800 \mathrm{pg} / \mathrm{ml}$ (normal reference values: $13-115 \mathrm{pg} / \mathrm{ml})$. Gastrin levels above 1000 $\mathrm{pg} / \mathrm{ml}$ were observed in $6(26.1 \%)$ patients.

\section{Incidence of positive tTG-Ab in different groups}

Five of 114 tTG-Ab tests were positive (4.2\%). All were females with Hashimoto's thyroiditis. Rates were equal among Jews and Arabs. One patient, a 61-year-old woman, was both PC-Ab and tTG-Ab positive.

\section{Relationship between thyroid antibodies, PC-Ab and tTG-AB}

The TPO-Ab level was significantly higher in patients with positive $\mathrm{PC}-\mathrm{Ab}$ compared to those without positive PC-Ab $(967 \pm 1371$ vs. $600.2 \pm 373$ respectively, $p=0.027)$. Tg-Ab levels were similar in patients with or without PC-Ab (Table I). Anti-thyroid antibody levels were not associated with positive tTG-Ab (Table II).

\section{Discussion}

The association of ATD with other autoimmune disorders is well established and is clinical- ly significant. However, routine measurement of PC-Ab and tTG-Ab is still not included in the clinical guidelines for evaluation and treatment of patients with ATD $[13,14]$.

\section{ATD and autoimmune gastritis}

This study demonstrates a $28.3 \%$ prevalence of PC-Ab in subjects with ATD, which is compatible with the published prevalence of $20-29 \%$ [2, $5,6]$. PC-Ab positivity is predictive of pathologic disturbances in gastric mucosa. PC-Ab predicted the development of chronic atrophic gastritis in a 5-year, prospective study of 208 adults with ATD [2]. In our series, $95.5 \%$ of the patients with positive PC-Ab who underwent an upper GI endoscopy had abnormal results. Atrophic gastritis was the major finding, documented in $73.1 \%$ of the endoscopies. A subgroup of $23.1 \%$ presented with intestinal metaplasia in addition to the atrophy, and $1(3.8 \%)$ patient presented with gastric NET type I. The other abnormal pathology results (23.1\%) were consistent with non-specific gastritis.

The loss of $\mathrm{HCl}$-secreting cells in the stomach secondary to the autoimmune process results in hypergastrinemia. Hypergastrinemia was documented in the majority of subjects with positive PC-Ab (78.3\%). Gastrin level was extremely elevated in some of these patients, measuring as high as $2800 \mathrm{pg} / \mathrm{ml}$ (normal reference values: 13-115 $\mathrm{pg} / \mathrm{ml}$ ). Six of the 23 gastrin measurements were above $1000 \mathrm{pg} / \mathrm{ml}$, a level compatible with gastrin-secreting tumor (Zollinger-Ellison syndrome). Clinical judgment and other confirmatory procedures are necessary to differentiate between gastrin-secreting tumor and hypergastrinemia secondary to autoimmune gastritis [15].

Hypergastrinemia stimulates enterochromaffin cell hyperplasia in the gastric mucosa in ATD patients, which can develop into type I gastric NET $[4,16]$. Although type I gastric NET is considered

Table I. Association between anti-thyroid auto-antibody levels and positive PC-Ab

\begin{tabular}{|lcc|}
\hline PC-Ab & TPO-Ab level, \pm SD [IU/ml] & Tg-Ab level, \pm SD [IU/ml] \\
\hline Positive & $967.06 \pm 1371.97(n=33)$ & $355.57 \pm 767.06(n=30)$ \\
\hline Negative & $600.24 \pm 373.80(n=82)$ & $552.2 \pm 1032.34(n=83)$ \\
\hline$P$-value (t-test for equality of means) & 0.027 & 0.343 \\
\hline$P C$-Ab-anti-parietal cell antibodies, TPO-Ab-anti-thyroid peroxidase antibodies, $T g$-Ab-anti-thyroglobulin antibodies.
\end{tabular}

Table II. Association between anti-thyroid auto-antibody levels and positive tTG-Ab

\begin{tabular}{|lcc|}
\hline tTG-Ab & TPO Ab level, \pm SD [IU/ml] & Tg-Ab level, \pm SD [IU $/ \mathrm{ml}]$ \\
\hline Positive & $463.50 \pm 353.77(n=4)$ & $785.5 \pm 1476.84(n=4)$ \\
\hline Negative & $646.1 \pm 375.02(n=106)$ & $501.54 \pm 971.64(n=104)$ \\
\hline$P$-value ( $t$-test for equality of means) & 0.340 & 0.574 \\
\hline tTG- $A b$ - anti-tissue transglutaminase IgA antibodies, TPO-Ab-anti-thyroid peroxidase antibodies.
\end{tabular}


a benign lesion, recent data suggest that up to $8 \%$ metastasize to local lymph nodes and even to the liver [17].

Nevertheless, PC-Ab are predictors of autoimmune gastritis, as well as predictive markers of subsequent metabolic and hematologic manifestations. Autoimmune atrophic gastritis may manifest as vitamin $B_{12}$ deficiency, pernicious anemia or iron deficiency anemia. Vitamin $B_{12}$ deficiency was detected in $28 \%$ of subjects with ATD [7] and was defined as the causative agent for anemia in $10 \%$ of subjects with hypothyroidism [18]. In addition to the aforementioned effects of autoimmune gastritis, PC-Ab positivity is associated with increased L-thyroxine requirements in hypothyroid subjects [19].

\section{ATD and celiac disease}

In this cohort, $4.2 \%$ of the subjects with ATD showed tTG-Ab positivity. This rate is compatible with that published in the literature $[10,11]$ and is much higher than the estimated prevalence in the general population, which is $0.3-1.4 \%$ in most countries [12]. Immunoglobulin A (IgA) tTG-Ab has high specificity and sensitivity for detection of celiac disease in adults and its level correlates closely with disease activity [20]. Early recognition of celiac disease enables prompt treatment with a gluten-free diet, which is associated with symptomatic improvement, reduced malignancy risk and a reduced mortality rate [11].

The study population included Arabs (20\%) and Jews (80\%). The rates of PC-Ab positivity and tTG$\mathrm{Ab}$ positivity in these ethnic groups were similar.

\section{Anti-thyroid antibodies, PC-Ab and tTG-Ab}

Another interesting finding was the relation between anti-thyroid Ab levels, PC-Ab and tTG-Ab. The TPO-Ab level was significantly higher in patients with positive PC-Ab, but not in those with positive tTG-Ab. Tg-Ab level was not associated with higher levels of other antibodies. Interestingly, the TPO-Ab/Tg-Ab ratio in PC-Ab positive patients was 2.71, while the ratio in tTG-Ab positive patients was 0.59 . The small sample size of tTGAb positive patients $(n=4)$ does not allow statistical analysis of these results. We are not aware of a specific role of the thyroid autoantibodies in the pathogenesis of either atrophic gastritis or celiac disease.

Although this study underscores the importance of PC-Ab and tTG-Ab measurements in patients with ATD, it has several potential limitations that should be considered. First, the data were collected retrospectively. However, the rates of PC-Ab and tTG-Ab positivity are similar to those published in other studies. Second, the study is lacking long-term patient follow-up and data concerning hematologic and metabolic status. Nevertheless, information about the prevalence of anemia and vitamin $B_{12}$ deficiency in this population has already been published [7].

In conclusion, the results of this study, combined with previously published data, support routine screening for PC-Ab and tTG-Ab in patients with ATD, especially those with high TPO-Ab levels.

\section{Conflict of interest}

The authors declare no conflict of interest.

\section{References}

1. Toh BH. Diagnosis and classification of autoimmune gastritis. Autoimmun Rev 2014; 13: 459-62.

2. Tozzoli R, Kodermaz G, Perosa AR, et al. Autoantibodies to parietal cells as predictors of atrophic body gastritis: a five-year prospective study in patients with autoimmune thyroid diseases. Autoimmun Rev 2010; 10: 80-3.

3. Uibo R, Krohn K, Villako K, Tammur R, Tamm A. The relationship of parietal cell, gastrin cell, and thyroid autoantibodies to the state of the gastric mucosa in a population sample. Scand J Gastroenterol 1984; 19: 1075-80.

4. Alexandraki KI, Nikolaou A, Thomas D, et al. Are patients with autoimmune thyroid disease and autoimmune gastritis at risk of gastric neuroendocrine neoplasms type 1? Clin Endocrinol (Oxf) 2014; 80: 685-90.

5. Checchi S, Montanaro A, Ciuoli C, et al. Prevalence of parietal cell antibodies in a large cohort of patients with autoimmune thyroiditis. Thyroid 2010; 20: 1385-9.

6. García García B, Gimeno Orna JA, Aguillo Gutiérrez E, et al. Prevalence and predictive factors of parietal cell antibody positivity in autoimmune thyroid disease [Spanish]. Endocrinol Nutr 2010; 57: 49-53.

7. Ness-Abramof R, Nabriski DA, Braverman LE, et al. Prevalence and evaluation of B12 deficiency in patients with autoimmune thyroid disease. Am J Med Sci 2006; 332: 119-22.

8. Rampertab SD, Pooran N, Brar P, Singh P, Green PH. Trends in the presentation of celiac disease. Am J Med 2006; 119: 355.e9-14.

9. Di Sabatino A, Vanoli A, Giuffrida P, Luinetti O, Solcia E, Corazza GR. The function of tissue transglutaminase in celiac disease. Autoimmun Rev 2012; 11: 746-53.

10. Meloni GF, Tomasi PA, Bertoncelli A, Fanciulli G, Delitala G, Meloni T. Prevalence of silent celiac disease in patients with autoimmune thyroiditis from Northern Sardinia. J Endocrinol Invest 2001; 24: 298-302.

11. Spadaccino AC, Basso D, Chiarelli S, et al. Celiac disease in North Italian patients with autoimmune thyroid diseases. Autoimmunity 2008; 41: 116-21.

12. Gujral N, Freeman HJ, Thomson AB. Celiac disease: prevalence, diagnosis, pathogenesis and treatment. World J Gastroenterol 2012; 18: 6036-59.

13. Garber JR, Cobin RH, Gharib H, et al.; American Association of Clinical Endocrinologists and American Thyroid Association Taskforce on Hypothyroidism in Adults. Clinical practice guidelines for hypothyroidism in adults: cosponsored by the American Association of Clinical Endocrinologists and the American Thyroid Association. Endocr Pract 2012; 18: 988-1028. 
14. Bahn Chair RS, Burch HB, Cooper DS, et al.; American Thyroid Association; American Association of Clinical Endocrinologists. Hyperthyroidism and other causes of thyrotoxicosis: management guidelines of the American Thyroid Association and American Association of Clinical Endocrinologists. Thyroid 2011; 21: 593-646. Erratum in: Thyroid 2011; 21: 1169. Thyroid 2012; 22: 1195.

15. Berna MJ, Hoffmann KM, Long SH, Serrano J, Gibril F, Jensen RT. Serum gastrin in Zollinger-Ellison syndrome: II. Prospective study of gastrin provocative testing in 293 patients from the National Institutes of Health and comparison with 537 cases from the literature. Evaluation of diagnostic criteria, proposal of new criteria, and correlations with clinical and tumoral features. Medicine (Baltimore) 2006; 85: 331-64.

16. Nicolaou A, Thomas D, Alexandraki KI, Sougioultzis S, Tsolakis AV, Kaltsas G. Predictive value of gastrin levels for the diagnosis of gastric enterochromaffin-like cell hyperplasia in patients with Hashimoto's thyroiditis. Neuroendocrinology 2014; 99: 118-22.

17. Grozinsky-Glasberg S, Thomas D, Strosberg JR, et al.; Metastatic type 1 gastric carcinoid: a real threat or just a myth? World J Gastroenterol 2013; 19: 8687-95.

18. Das C, Sahana PK, Sengupta N, Giri D, Roy M, Mukhopadhyay P. Etiology of anemia in primary hypothyroid subjects in a tertiary care center in Eastern India. Indian J Endocrinol Metab 2012; 16 (Suppl. 2): S361-3.

19. Checchi S, Montanaro A, Pasqui L, et al. L-thyroxine requirement in patients with autoimmune hypothyroidism and parietal cell antibodies. J Clin Endocrinol Metab 2008; 93: 465-9.

20. Osman M, Taha B, Al Duboni G. Assessment of the response to gluten-free diet in an Iraqi population with coeliac disease. A histological and serological follow-up study. Arch Med Sci 2014; 10: 294-9. 